ITP-UH-06/00

hep-th/0005044

\title{
New $\mathbf{N}=2$ Supersymmetric Vector-Tensor Interaction
}

\author{
Ulrich Theis \\ Institut für Theoretische Physik, Universität Hannover, \\ Appelstraße 2, 30167 Hannover, Germany \\ utheis@itp.uni-hannover.de
}

\begin{abstract}
An $N=2$ supersymmetric self-interaction of the vector-tensor multiplet is presented, in which the vector provides the gauge field for local central charge transformations. The dual description in terms of a vector multiplet and an $N=1$ superspace formulation are given.
\end{abstract}

Since the discovery of the relevance of the $N=2$ vector-tensor multiplet [1] to certain heterotic string theory compactifications [2], its possible interactions have been investi-

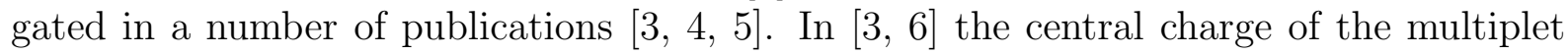
was gauged by a coupling to an abelian vector multiplet that provides the gauge field for local central charge transformations, while in [- 1 a self-interaction of the multiplet was introduced, giving rise to a Chern-Simons coupling of the vector and tensor. These two kinds of interactions have subsequently been rederived in harmonic superspace from deformations of the superfield constraints that determine the multiplet [7, 8].

In the present letter it is shown how the central charge of the vector-tensor multiplet can be gauged by a self-interaction, without a coupling to an additional vector multiplet. The model is obtained from a deformation of the free component action and its corresponding supersymmetry and gauge transformations by means of the Noether method. This results in a realization of the supersymmetry algebra which holds only on-shell, i.e. on fields satisfying their equations of motion. We shall address the problem of constructing a manifestly $N=2$ supersymmetric off-shell formulation in a future publication and content ourselves with an off-shell version in terms of $N=1$ superfields for the time being.

Gauge field theories of the kind considered here have been investigated in detail in [9] in the framework of $N=1$ supersymmetry. They are actually members of a family of (bosonic) models discovered by Henneaux and Knaepen in [10], that in four spacetime dimensions involve non-polynomial interactions of 1 - and 2-forms. In [9] it was shown that every four-dimensional Henneaux-Knaepen model admits an $N=1$ supersymmetric generalization. The vector-tensor multiplet with gauged central charge as found in [3] was so far the only known model with two supersymmetries. In the following we present the second example. 
The vector-tensor multiplet contains a 1 -form $A_{\mu}$, a 2 -form $B_{\mu \nu}$, a real scalar $\phi$, and an $\mathrm{SU}(2)$ doublet of Weyl spinors $\psi_{\alpha}^{i}$. The bosonic and fermionic off-shell degrees of freedom can be matched by inclusion of a real auxiliary scalar $U$.

The supersymmetry algebra of the free multiplet involves a central charge that acts nontrivially on the gauge fields,

$$
\Delta_{c} A_{\mu}=c H_{\mu}, \quad \Delta_{c} B_{\mu \nu}=c * F_{\mu \nu}, \quad c \in \mathbb{R} .
$$

Here $* F^{\mu \nu}=\varepsilon^{\mu \nu \rho \sigma} \partial_{\rho} A_{\sigma}$ and $H^{\mu}=\frac{1}{2} \varepsilon^{\mu \nu \rho \sigma} \partial_{\nu} B_{\rho \sigma}$ are the Hodge-dual field strengths of $A_{\mu}$ and $B_{\mu \nu}$ respectively. The conserved current that corresponds to this global symmetry of the free action is simply

$$
J^{\mu}=F^{\mu \nu} H_{\nu}
$$

A coupling of $A_{\mu}$ to this current yields a consistent interaction vertex $g A_{\mu} J^{\mu}$ of dimension five (where accordingly $g$ is a coupling constant of dimension -1 ). It requires to modify the gauge transformations of $A_{\mu}$ and $B_{\mu \nu}$ by first-order terms that are precisely the central charge transformations given above,

$$
\begin{aligned}
\delta A_{\mu} & =\partial_{\mu} C(x)+g C(x) H_{\mu}+O\left(g^{2}\right) \\
\delta B_{\mu \nu} & =\partial_{\mu} C_{\nu}(x)-\partial_{\nu} C_{\mu}(x)+g C(x) * F_{\mu \nu}+O\left(g^{2}\right),
\end{aligned}
$$

which amounts to gauging the global symmetry. In order to restore supersymmetry to order $g$, the vertex $A_{\mu} J^{\mu}$ is to be accompanied by additional terms of dimension five, which however depend on the gauge potentials only via the field strengths and therefore do not introduce further first-order deformations of the gauge transformations. The question now is whether the action and the transformations can be extended such that both gauge invariance and $N=2$ supersymmetry are realized to all orders in the coupling constant. Since the interaction falls into the family of Henneaux-Knaepen models, it is to be expected that the resulting action and transformations are non-polynomial in the fields, so a Noether construction order by order seems impractical. As it turns out, however, the introduction of an auxiliary vector $V^{\mu}$, which to lowest order in $g$ equals $H^{\mu}$ on-shell, greatly simplifies the construction: it essentially suffices to determine the deformation up to second order, all higher-order contributions are due to prefactors that are functions of the scalar field $\phi$ only, which are easily completed to all orders.

The full Lagrangian constructed in this way reads

$$
\begin{aligned}
\mathcal{L}= & \frac{1}{2} \cos (2 g \phi) V^{\mu} V_{\mu}-V_{\mu} H^{\mu}-\frac{1}{4} \cos (2 g \phi) \mathcal{F}^{\mu \nu} \mathcal{F}_{\mu \nu}-\frac{1}{4} \sin (2 g \phi) * \mathcal{F}^{\mu \nu} \mathcal{F}_{\mu \nu} \\
& +\frac{1}{2} \cos (2 g \phi) \partial^{\mu} \phi \partial_{\mu} \phi-\mathrm{i} \cos (2 g \phi)\left(\psi^{i} \sigma^{\mu} \stackrel{\leftrightarrow}{\partial_{\mu}} \bar{\psi}_{i}\right)+2 g \sin (2 g \phi) V_{\mu} \psi^{i} \sigma^{\mu} \bar{\psi}_{i}+\frac{1}{2} U^{2} \\
& -\mathrm{i} g \mathcal{F}_{\mu \nu}\left(\mathrm{e}^{\mathrm{i} g \phi} \psi^{i} \sigma^{\mu \nu} \psi_{i}-\mathrm{e}^{-\mathrm{i} g \phi} \bar{\psi}^{i} \bar{\sigma}^{\mu \nu} \bar{\psi}_{i}\right)-\frac{g^{2}}{\cos (2 g \phi)}\left(\mathrm{e}^{-\mathrm{i} g \phi} \psi^{i} \psi^{j}+\mathrm{e}^{\mathrm{i} g \phi} \bar{\psi}^{i} \bar{\psi}^{j}\right)^{2} .
\end{aligned}
$$

Here $\mathcal{F}_{\mu \nu}$ is an extended field strength,

$$
\mathcal{F}_{\mu \nu}=\left(\partial_{\mu}+g V_{\mu}\right) A_{\nu}-\left(\partial_{\nu}+g V_{\nu}\right) A_{\mu}
$$


The Lagrangian is invariant, up to a total derivative, under gauge transformations

$$
\begin{aligned}
\delta A_{\mu}= & \left(\partial_{\mu}+g V_{\mu}\right) C \\
\delta B_{\mu \nu}= & g C\left[\cos (2 g \phi) * \mathcal{F}_{\mu \nu}-\sin (2 g \phi) \mathcal{F}_{\mu \nu}-2 g\left(\mathrm{e}^{\mathrm{i} g \phi} \psi^{i} \sigma_{\mu \nu} \psi_{i}+\mathrm{e}^{-\mathrm{i} g \phi} \bar{\psi}^{i} \bar{\sigma}_{\mu \nu} \bar{\psi}_{i}\right)\right] \\
& +\partial_{\mu} C_{\nu}-\partial_{\nu} C_{\mu} \\
\delta \phi= & \delta \psi_{\alpha}^{i}=\delta V_{\mu}=\delta U=0,
\end{aligned}
$$

and rigid supersymmetry transformations generated by

$$
\begin{aligned}
\mathcal{D}_{\alpha}^{i} \phi= & \psi_{\alpha}^{i} \\
\mathcal{D}_{\alpha}^{i} V_{\mu}= & -\mathrm{i} \partial_{\mu} \psi_{\alpha}^{i} \\
\mathcal{D}_{\alpha}^{i} A_{\mu}= & \mathrm{i} g \psi_{\alpha}^{i} A_{\mu}+\mathrm{i} \mathrm{e}^{\mathrm{i} g \phi}\left(\sigma_{\mu} \bar{\psi}^{i}\right)_{\alpha} \\
\mathcal{D}_{\alpha}^{i} B_{\mu \nu}= & 2 \cos (2 g \phi)\left(\sigma_{\mu \nu} \psi^{i}\right)_{\alpha}-2 g \mathrm{e}^{-\mathrm{i} g \phi} A_{[\mu}\left(\sigma_{\nu]} \bar{\psi}^{i}\right)_{\alpha} \\
\mathcal{D}_{\alpha}^{i} \bar{\psi}_{\dot{\alpha}}^{j}= & \frac{1}{2} \varepsilon^{i j} \sigma_{\alpha \dot{\alpha}}^{\mu}\left(V_{\mu}+\mathrm{i} \partial_{\mu} \phi\right) \\
\mathcal{D}_{\alpha}^{i} \psi^{\beta j}= & \frac{1}{2} \varepsilon^{i j} \mathrm{e}^{\mathrm{i} g \phi} \mathcal{F}_{\mu \nu} \sigma_{\alpha \nu}^{\mu \nu}+\frac{\mathrm{i}}{2} \varepsilon^{i j} \delta_{\alpha}^{\beta} U-\mathrm{i} g \varepsilon^{i j} \psi_{\alpha}^{k} \psi_{k}^{\beta}-g \tan (2 g \phi) \delta_{\alpha}^{\beta} \psi^{i} \psi^{j} \\
& -\frac{\mathrm{i} g}{\cos (2 g \phi)} \delta_{\alpha}^{\beta} \bar{\psi}^{i} \bar{\psi}^{j} \\
\mathcal{D}_{\alpha}^{i} U= & -\cos (2 g \phi)\left(\sigma^{\mu} \partial_{\mu} \bar{\psi}^{i}\right)_{\alpha}+g \sin (2 g \phi)\left(\partial_{\mu} \phi-\mathrm{i} V_{\mu}\right)\left(\sigma^{\mu} \bar{\psi}^{i}\right)_{\alpha} \\
& -g \mathrm{e}^{\mathrm{i} g \phi} \mathcal{F}_{\mu \nu}\left(\sigma^{\mu \nu} \psi^{i}\right)_{\alpha}-\frac{2 \mathrm{i} g^{2}}{\cos (2 g \phi)}\left(\mathrm{e}^{-2 \mathrm{i} g \phi} \psi^{i} \psi^{j}+\bar{\psi}^{i} \bar{\psi}^{j}\right) \psi_{\alpha j} .
\end{aligned}
$$

Using the transformation properties of $\mathcal{F}_{\mu \nu}$,

$$
\begin{aligned}
\delta \mathcal{F}_{\mu \nu} & =g C\left(\partial_{\mu} V_{\nu}-\partial_{\nu} V_{\mu}\right) \\
\mathcal{D}_{\alpha}^{i} \mathcal{F}_{\mu \nu} & =\mathrm{i} g \psi_{\alpha}^{i} \mathcal{F}_{\mu \nu}+2 \mathrm{i}\left(\partial_{[\mu}+g V_{[\mu}\right)\left(\mathrm{e}^{\mathrm{i} g \phi} \sigma_{\nu]} \bar{\psi}^{i}\right)_{\alpha},
\end{aligned}
$$

gauge invariance is easily verified, while supersymmetry requires some effort.

Note that although the combination $\partial_{\mu}+g V_{\mu}$ resembles a covariant derivative, there is no gauge transformation associated with $V_{\mu}$, it is merely an auxiliary field. In order to eliminate it, we collect all terms involving $V_{\mu}$. They can be written as

$$
\mathcal{L}=\frac{1}{2} V_{\mu} K^{\mu \nu} V_{\nu}-V_{\mu} \mathcal{H}^{\mu}+\ldots
$$

where we have employed the abbreviations

$$
\begin{aligned}
K^{\mu \nu}= & \cos (2 g \phi)\left[\eta^{\mu \nu}\left(1-g^{2} A \cdot A\right)+g^{2} A^{\mu} A^{\nu}\right] \\
\mathcal{H}^{\mu}= & \frac{1}{2} \varepsilon^{\mu \nu \rho \sigma} \partial_{\nu} B_{\rho \sigma}+g \cos (2 g \phi) F^{\mu \nu} A_{\nu}+g \sin (2 g \phi) * F^{\mu \nu} A_{\nu} \\
& -2 g \sin (2 g \phi) \psi^{i} \sigma^{\mu} \bar{\psi}_{i}+2 \mathrm{i} g^{2}\left(\mathrm{e}^{\mathrm{i} g \phi} \psi^{i} \sigma^{\mu \nu} \psi_{i}-\mathrm{e}^{-\mathrm{i} g \phi} \bar{\psi}^{i} \bar{\sigma}^{\mu \nu} \bar{\psi}_{i}\right) A_{\nu} .
\end{aligned}
$$

The equation of motion of $V_{\mu}$ then yields ( $\approx$ denotes on-shell equality)

$$
V_{\mu} \approx\left(K^{-1}\right)_{\mu \nu} \mathcal{H}^{\nu}, \quad\left(K^{-1}\right)_{\mu \nu}=\frac{\eta_{\mu \nu}-g^{2} A_{\mu} A_{\nu}}{\cos (2 g \phi)\left(1-g^{2} A \cdot A\right)},
$$


which upon insertion into the Lagrangian and the transformations results in expressions non-polynomial in both $g A_{\mu}$ and $g \phi$. If one expands the Lagrangian in powers of $g$, the coupling $g A_{\mu} J^{\mu}$ is recovered to first order, which was the goal of the construction.

The supersymmetry algebra closes on-shell; if the equations of motion hold, the commutator of two supersymmetry transformations $\Delta_{\xi}=\xi_{i}^{\alpha} \mathcal{D}_{\alpha}^{i}+\bar{\xi}_{\dot{\alpha}}^{i} \overline{\mathcal{D}}_{i}^{\dot{\alpha}}$ yields a translation and a gauge transformation,

$$
\left[\Delta_{\xi}, \Delta_{\zeta}\right] \approx a^{\mu} \partial_{\mu}-\delta
$$

with parameters

$$
\begin{aligned}
a^{\mu} & =\mathrm{i}\left(\zeta_{i} \sigma^{\mu} \bar{\xi}^{i}-\xi_{i} \sigma^{\mu} \bar{\zeta}^{i}\right) \\
C & =a^{\mu} A_{\mu}+\frac{\mathrm{i}}{g}\left(\mathrm{e}^{\mathrm{i} g \phi} \xi_{i} \zeta^{i}+\mathrm{e}^{-\mathrm{i} g \phi} \bar{\xi}^{i} \bar{\zeta}_{i}\right) \\
C_{\mu} & =a^{\nu} B_{\nu \mu}+\frac{1}{2 g} \sin (2 g \phi) a_{\mu}+\left(\mathrm{e}^{\mathrm{i} g \phi} \bar{\xi}^{i} \bar{\zeta}_{i}-\mathrm{e}^{-\mathrm{i} g \phi} \xi_{i} \zeta^{i}\right) A_{\mu} .
\end{aligned}
$$

Note that while the parameter $C$ is singular for $g=0$, the transformation $\delta$ is not, since $C$ occurs either under a derivative or with a factor $g$.

It is quite remarkable that the gauged central charge transformations do not commute with supersymmetry transformations, not even on-shell. Rather, one has

$$
\left[\Delta_{\xi}, \delta\right] \approx \delta^{\prime}
$$

where now

$$
\begin{aligned}
& C^{\prime}=\mathrm{i} g C\left(\xi_{i} \psi^{i}-\bar{\xi}^{i} \bar{\psi}_{i}\right) \\
& C_{\mu}^{\prime}=g C\left(\mathrm{e}^{-\mathrm{i} g \phi} \xi^{i} \sigma_{\mu} \bar{\psi}_{i}-\mathrm{e}^{\mathrm{i} g \phi} \psi^{i} \sigma_{\mu} \bar{\xi}_{i}\right) .
\end{aligned}
$$

A free vector-tensor multiplet is dual to an abelian vector multiplet. Since in our interacting model the 2 -form $B_{\mu \nu}$ occurs only via its field strength, it too can be dualized into a pseudo-scalar. In fact, the use of an auxiliary vector makes the dualization particularly simple. Considering $H^{\mu}$ as an independent field and implementing the Bianchi identity $\partial_{\mu} H^{\mu}=0$ by means of a Lagrange multiplier field $\varphi$, we find that according to the equation of motion of $H^{\mu}$, we can replace $V_{\mu}$ with $\partial_{\mu} \varphi$,

$$
V_{\mu} \rightarrow \partial_{\mu} \varphi, \quad \mathcal{D}_{\alpha}^{i} \varphi=-\mathrm{i} \psi_{\alpha}^{i} .
$$

$\phi$ and $\varphi$ can be combined into a complex scalar $X$ that is chiral, $\overline{\mathcal{D}}_{\dot{\alpha} i} X=0$. With suitable field redefintions, we obtain the (on-shell) field content of an $N=2$ vector multiplet with the standard supersymmetry transformations",

$$
X=\frac{1}{2 g} \mathrm{e}^{g(\varphi-\mathrm{i} \phi)}, \quad \hat{A}_{\mu}=\mathrm{e}^{g \varphi} A_{\mu}, \quad \lambda_{\alpha}^{i}=\mathrm{e}^{g(\varphi-\mathrm{i} \phi)} \psi_{\alpha}^{i} .
$$

\footnotetext{
${ }^{1}$ Every function of $(\varphi-\mathrm{i} \phi)$ is chiral, but only $X$ as in 17 ) satisfies the additional constraint $\mathcal{D}^{i} \mathcal{D}^{j} X+$ $\overline{\mathcal{D}}^{i} \overline{\mathcal{D}}^{j} \bar{X}=0$ that gives rise to a vector multiplet.
} 
Up to a factor, $\mathcal{F}_{\mu \nu}$ turns out to be nothing but the field strength of the new vector $\hat{A}_{\mu}$,

$$
\mathcal{F}_{\mu \nu}=\mathrm{e}^{-g \varphi} \hat{F}_{\mu \nu} .
$$

The holomorphic prepotential $f(X)$ that determines the action of the vector multiplet can be obtained from the field strength terms in the Lagrangian (四),

$$
-\frac{1}{4} \cos (2 g \phi) \mathcal{F}^{\mu \nu} \mathcal{F}_{\mu \nu}-\frac{1}{4} \sin (2 g \phi) * \mathcal{F}^{\mu \nu} \mathcal{F}_{\mu \nu}=-\frac{1}{4} \operatorname{Im}\left[\frac{\mathrm{i}}{(2 g X)^{2}} \hat{F}_{\mu \nu}\left(\hat{F}^{\mu \nu}-\mathrm{i} * \hat{F}^{\mu \nu}\right)\right] .
$$

Comparing with the general expression common to every vector multiplet,

$$
-\frac{1}{4} \operatorname{Im}\left[f^{\prime \prime}(X) \hat{F}_{\mu \nu}\left(\hat{F}^{\mu \nu}-\mathrm{i} * \hat{F}^{\mu \nu}\right)\right],
$$

we read off the second derivative of the prepotential, and conclude that

$$
f(X)=-\frac{\mathrm{i}}{4 g^{2}} \ln (g X) .
$$

Finally, we briefly demonstrate how the present model can be derived from an $N=1$ superspace integral, drawing on results obtained in [9]:

Let us embed $B_{\mu \nu}, \phi$ and $\psi_{\alpha}^{1}$ in a chiral spinor superfield $\Psi_{\alpha}(x, \theta, \bar{\theta}), A_{\mu}$ and $\psi_{\alpha}^{2}$ in a real superfield $A(x, \theta, \bar{\theta})$, and $V_{\mu}$ in a real superfield $V(x, \theta, \bar{\theta})$ with a mass dimension shifted by +1 . From these, we construct field strength superfields $Y_{\alpha}(x, \theta, \bar{\theta})$ and $W_{\alpha}(x, \theta, \bar{\theta})$,

$$
Y_{\alpha}=-\frac{\mathrm{i}}{4} \bar{D}^{2}\left[\mathrm{e}^{-2 \mathrm{i} g V} D_{\alpha}\left(\mathrm{e}^{\mathrm{i} g V} A\right)\right], \quad W_{\alpha}=\frac{1}{2} \bar{D}^{2} D_{\alpha} V,
$$

where $D_{\alpha}$ and $\bar{D}_{\dot{\alpha}}$ are the usual $N=1$ supercovariant derivatives. $\Psi_{\alpha}, Y_{\alpha}$ and $W_{\alpha}$ are chiral,

$$
\bar{D}_{\dot{\alpha}} \Psi_{\alpha}=\bar{D}_{\dot{\alpha}} Y_{\alpha}=\bar{D}_{\dot{\alpha}} W_{\alpha}=0,
$$

so the action

$$
S=\int d^{4} x d^{2} \theta\left(W^{\alpha} \Psi_{\alpha}+Y^{\alpha} Y_{\alpha}-\frac{1}{4 g^{2}} d^{2} \bar{\theta} \mathrm{e}^{2 \mathrm{i} g V}\right)+\text { c.c. }
$$

is manifestly $N=1$ supersymmetric. The component gauge transformations (6) follow from the superfield transformations

$$
\delta \Psi_{\alpha}=\mathrm{i} \bar{D}^{2} D_{\alpha} R-2 \mathrm{i} g \Lambda Y_{\alpha}, \quad \delta A=\mathrm{i}\left(\mathrm{e}^{\mathrm{i} g V} \Lambda-\mathrm{e}^{-\mathrm{i} g V} \bar{\Lambda}\right), \quad \delta V=0 ，
$$

where $R(x, \theta, \bar{\theta})$ is a real and $\Lambda(x, \theta, \bar{\theta})$ a chiral superfield. Gauge invariance of the action is then due to the relations

$$
\delta Y_{\alpha}=\mathrm{i} g \Lambda W_{\alpha}, \quad D^{\alpha} W_{\alpha}-\bar{D}_{\dot{\alpha}} \bar{W}^{\dot{\alpha}}=0,
$$


the former being the superfield analog of the gauge transformation (8) of $\mathcal{F}_{\mu \nu}$. Passing to Wess-Zumino gauge for $A$ and $\Psi_{\alpha}$ and eliminating the auxiliary fields (except $V_{\mu}$ ) results in the $N=2$ supersymmetric Lagrangian (4).

Evidently, there is an infinite number of $N=1$ supersymmetric Henneaux-Knaepen models that describe the field content of a vector-tensor multiplet, for one may specify different functions of $V$ (the $d^{2} \theta d^{2} \bar{\theta}$-part in the action) that allow to eliminate the auxiliary fields (another example was given in [9]), but in general these will not possess a second supersymmetry.

The previously known interactions of the vector-tensor multiplet can all be derived by dimensional reduction from interactions of the $N=(1,0)$ tensor multiplet in six dimensions [11, 8]. In these cases the gauged central charge is a remnant of translations in the additional spacelike directions. It would be interesting to know whether this applies to the present model as well, or whether the fact that the gauge field for the local central charge transformations resides in the vector-tensor multiplet itself requires a different mechanism in six dimensions. Models that might be relevant in this context can be found in [12].

The new model introduced here indicates that our current knowledge about the possible interactions of the vector-tensor multiplet is far from being exhaustive. In particular, the problem of finding interactions between several such multiplets has not been attacked successfully as yet. A possible starting point would be to consider the general $N=1$ supersymmetric Henneaux-Knaepen models of [9] and to single out those that exhibit an $\mathrm{SU}(2) R$-symmetry. Work in this direction is under way.

Remark: In the course of this work, a further $N=2$ supersymmetric Henneaux-Knaepen model was found, involving two vector multiplets and a double-tensor multiplet [13].

\section{Acknowledgements}

I would like to thank Friedemann Brandt and Sergei Kuzenko for helpful discussions and suggestions. Thanks also to Augusto Sagnotti for bringing the work on six-dimensional tensor multiplet couplings to my attention.

\section{References}

[1] M.F. Sohnius, K.S. Stelle and P.C. West, Phys. Lett. B 92 (1980) 123.

[2] B. de Wit, V. Kaplunovsky, J. Louis and D. Lüst, Nucl. Phys. B 451 (1995) 53 (hepth/9504006).

[3] P. Claus, B. de Wit, M. Faux, B. Kleijn, R. Siebelink and P. Termonia, Phys. Lett. B 373 (1996) 81 hep-th/9512143.

[4] P. Claus, B. de Wit, M. Faux and P. Termonia, Nucl. Phys. B 491 (1997) 201 (hepth/9612203).

[5] R. Grimm, M. Hasler and C. Herrmann, Int. J. Mod. Phys. A 13 (1998) 1805 (hepth/9706108);

N. Dragon, S. Kuzenko and U. Theis, Eur. Phys. J. C 4 (1998) 717 (hep-th/9706169); 
I. Buchbinder, A. Hindawi and B.A. Ovrut, Phys. Lett. B 413 (1997) 79 (hep-th/9706216); P. Claus, B. de Wit, M. Faux, B. Kleijn, R. Siebelink and P. Termonia, Nucl. Phys. B 512 (1998) 148 (hep-th/9710212).

[6] N. Dragon and U. Theis, Phys. Lett. B 446 (1999) 314 (hep-th/9805199).

[7] N. Dragon and S. Kuzenko, Phys. Lett. B 420 (1998) 64 (hep-th/9709088);

E. Ivanov and E. Sokatchev, Phys. Lett. B 429 (1998) 35 (hep-th/9711038).

[8] N. Dragon, E. Ivanov, S. Kuzenko, E. Sokatchev and U. Theis, Nucl. Phys. B 538 (1999) 411 (hep-th/9805152).

[9] F. Brandt and U. Theis, Nucl. Phys. B 550 (1999) 495 (hep-th/9811180).

[10] M. Henneaux and B. Knaepen, Phys. Rev. D 56 (1997) 6076 (hep-th/9706119);

M. Henneaux and B. Knaepen, A theorem on first-order interaction vertices for free $p$-form gauge fields. Preprint ULB-TH-99/30, DAMTP-1999-172 (hep-th/9912052).

[11] E. Bergshoeff, E. Sezgin and A. van Proeyen, Nucl. Phys. B 264 (1986) 653;

E. Bergshoeff, E. Sezgin and E. Sokatchev, Class. Quantum Grav. 13 (1996) 2875 (hepth/9605087).

[12] H. Nishino and E. Sezgin, Nucl. Phys. B 505 (1997) 497 (hep-th/9703075);

G. Dall'Agata and K. Lechner, Nucl. Phys. B 511 (1998) 326 (hep-th/9707236);

S. Ferrara, F. Riccioni and A. Sagnotti, Nucl. Phys. B 519 (1998) 115 (hep-th/9711059);

F. Riccioni and A. Sagnotti, Phys. Lett. B 436 (1998) 298 (hep-th/9806129).

[13] F. Brandt, New N=2 supersymmetric gauge theories: the double tensor multiplet and its interactions. Preprint ITP-UH-07/00 (hep-th/0005086). 